\title{
Simposio \\ ENFERMEDADES PARASITARIAS \\ DE LA CUENCA AMAZÓNICA
}

\section{Eliminación de la oncocercosis en Ecuador: un sueño hecho realidad}

\author{
Mauricio Espinel \\ Universidad San Francisco de Quito, Quito, Ecuador
}

Sendas publicaciones sobre la situación epidemiológica de la oncocercosis en el Ecuador se realizaron en las décadas del 80 y el 90 , describiendo con mucha amplitud un foco de transmisión en el norte de la costa del Ecuador, en la provincia de Esmeraldas, la epidemiología, las manifestaciones clínicas, los aspectos inmunológicos, oftalmológicos y dermatológicos, y la integración con programas de atención primaria, etc. Se han publicado más de 70 artículos científicos en revistas internacionales, que son prueba evidente de cómo un programa de investigación puede ser el eje de un programa de control.

La zona en mención es una de las de mayor riqueza en biodiversidad del país en la que, en un ambiente tropical, habitan comunidades afroecuatorianas e indígenas. Al mismo tiempo, es una zona con indicadores socioeconómicos de gran pobreza y marginación de los servicios básicos y sociales. En la zona existen 110 comunidades a lo largo de los ríos Cayapas, Santiago y Onzole, muchas de ellas de muy difícil acceso por la vía fluvial, con cerca de 40.000 habitantes.

Se encontraron comunidades con más de $90 \%$ de la población infectada, con lesiones oculares y de piel, y muchas otras complicaciones. Los estudios entomológicos encontraron como vector a Simulium exiguum, una mosca muy eficiente en la transmisión.

En este marco se inició en 1982 el Programa de Distribución de Ivermectina. Para lograr la meta de eliminación de la oncocercosis, se planteó como estándar una cobertura mayor de $90 \%$ de la población, para lo cual fue necesario involucrar a las comunidades y a todos los trabajadores de la salud en todo el proceso del control de la enfermedad.

El medicamento de elección para la oncocercosis es la ivermectina (Mectizan®), el cual elimina las microfilarias y disminuye su número en la piel por un lapso de seis meses, sin destruir los gusanos adultos. Este medicamento se administra en ciclos de seis meses por 12 a 15 años a, mínimo, 90 \% de las personas elegibles en cada comunidad, lo que conduce a la supresión de la transmisión. El tratamiento debe continuarse en igual forma durante 12 años, hasta lograr la eliminación de las manifestaciones de la enfermedad y la desaparición del parásito.

Al terminar la fase anterior, se suspende el tratamiento y se continúa con la fase de precertificación, que consiste en una vigilancia activa durante un período de tres años. Si no se detecta ninguna transmisión, se certifica la eliminación de la enfermedad en el país y se adquiere la condición posendémica.

Ecuador logró llegar a esta fase gracias al trabajo de los promotores y auxiliares de salud, y a un proceso de participación y acción muy interesante. Al momento se están iniciando las acciones de certificación de la eliminación.

El programa constituye un hito y un ejemplo para la salud pública del Ecuador. Es interesante cómo la confluencia de intereses académicos de investigación con los intereses de control de una enfermedad, puede lograr una situación de éxito. Éste es el caso de la oncocercosis en el Ecuador, en el que se logró un importante desarrollo académico en la investigación, se formó un importante número de investigadores y un equipo de trabajo de campo con amplias capacidades, y además, hubo una importante participación comunitaria, con formación y acción de promotores de salud de la zona y la integración de programas como el del control del niño sano y la embarazada.

Se debería impulsar este tipo de modelos, para lograr, por una parte, el detalle y la minuciosidad que busca la investigación y, por otra, las metas macrosociales que buscan los servicios de salud.

Bibliografía

1. Cooper PJ, Mancero T, Espinel M, et al. Early human infection with Onchocerca volvulus is associated 
with an enhanced parasite-specific cellular immune response. J Infect Dis. 2001;183:1662-8.

2. Cooper PJ, Proaño R, Beltrán C, Anselmi M, Guderian RH. Onchocerciasis in Ecuador: Changes in prevalence of ocular lesions in Onchocerca volvulus infected individuals over the period 1980-1990. Mem Inst Oswaldo Cruz. 1996;91:153-8.

3. Cupp EW, Duke BO, Mackenzie CD, Guzmán JR, Vieira JC, Méndez-Galvan J, et al. The effects of long-term community level treatment with ivermectin (Mectizan) on adult Onchocerca volvulus in Latin America. Am J Trop Med Hyg. 2004;71:602-7.

4. Espinel M. Onchocerciasis: A Latin American perspective. Ann Trop Med Parasitol. 1998; 92 (Suppl.1):S157-60.

5. Guderian JR, Anselmi M, Espinel M, Sandoval C, et al. Onchocerciasis in Ecuador: Prevalence of infection on the Ecuador-Colombia border in the Province of Esmeraldas. Mem Inst Oswaldo Cruz. 1997;92:15762.

6. Guderian RH, Anselmi M, Espinel M, Mancero T, et al. Successful control of onchocerciasis with communitybased ivermectin distribution in the Rio Santiago focus in Ecuador. Trop Med Int Health. 1997;2:982-8.

7. Guderian $\mathrm{RH}$, Kerrigan KR. Onchocerciasis and acquired groin hernias in Ecuador. Trop Med Parasitol. 1990 Mar;41(1):69-70.
8. Guderian RH, Lovato R, Anselmi M, Mancero T, Cooper PJ. Onchocerciasis and reproductive health in Ecuador. Trans R Soc Trop Med Hyg. 1997;91:315-7.

9. Guderian $\mathrm{RH}$, Molea J, Carrillo R. Onchocerciasis in Ecuador. III. Clinical manifestations of the disease in the province of Esmeraldas. Trans R Soc Trop Med Hyg. 1984;78:81-5.

10. Guderian RH, Molea J, Swanson D, et al. Onchocerciasis in Ecuador. I. Prevalence and distribution in the province of Esmeraldas. Tropenmed Parasitol. 1983;34:143-8.

11. Guderian RH, Shelley AJ. Onchocerciasis in Ecuador: The situation in 1989. Mem Inst Oswaldo Cruz. 1992;87:405-15.

12. Guderian RH, Swanson D, Carrillo R, et al. Onchocerciasis in Ecuador. II. Epidemiology of the endemic foci in the province of Esmeraldas. Tropenmed Parasitol. 1983;34:149-54.

13. Horton J, Witt C, Ottesen EA, Espinel M, et al. An analysis of the safety of the single dose, two drug regimens used in programmes to eliminate lymphatic filariasis. Parasitology. 2000;121(Suppl):S147-60.

14. Vieira JC, Cooper PJ, Lovato R, Mancero T, Rivera $J$, Proaño R, et al. Impact of long-term treatment of onchocerciasis with ivermectin in Ecuador: Potential for elimination of infection. BMC Med. 2007;23:9.

\title{
Enfermedad de Chagas en la región amazónica: la última frontera
}

\author{
José Rodrigues Coura, Angela Junqueir \\ Laboratório de Doenças Parasitárias (Medicina Tropical), Instituto Oswaldo Cruz, \\ Rio de Janeiro, RJ, Brasil
}

La región amazónica es la última frontera en la cual la enfermedad de Chagas aún no se ha vuelto endémica en América Latina. La región representa $44 \%$ del territorio suramericano y corresponde a los siguientes países, que tienen diferentes porcentajes de territorio amazónico: Bolivia $(35,61$ $\%)$, Brasil $(57,23 \%)$, Colombia (44,28\%), Ecuador $(47,05 \%)$, Guyana $(97,00 \%)$, Guyana Francesa (100\%), Perú $(59,45 \%)$, Surinam (100\%) y Venezuela (19,18\%) (Aguilar, et al., 2007).

La enfermedad de Chagas en la región amazónica como un todo, puede considerarse una enzootia de animales silvestres, dado que en muy pocos lugares se han encontrado focos de preadaptación o adaptación de triatominos en el domicilio o el peridomicilio humanos, como es el caso de Triatoma maculata y Panstrongylus geniculatus en la Amazonia brasilera, Rhodnius stalien el Alto Beni en Bolivia, y Rhodnius prolixus en el Orinoco, en la transición amazónica (Aguilar, et al., 2007), donde utilizan como alimentación animales domésticos, aves y animales silvestres como los marsupiales, que se aproximan al peridomicilio o invaden el domicilio humano en busca de alimentos.

Respecto a la infección humana, podemos considerar a la enfermedad de Chagas en la región amazónica como una antropozoonosis, o sea, una enfermedad accidental del hombre cuando éste penetra al ecosistema silvestre para pesca, caza, extracción vegetal, turismo o actividades ilegales, entre otras, o cuando los triatominos silvestres invaden el domicilio humano atraídos por la luz o en busca de alimentación con sangre humana a falta de mamíferos silvestres (Junqueira, et al., 2005). La transmisión oral por ingestión de alimentos contaminados por heces de triatominos, y orina o secreciones odoríferas de marsupiales, es de gran importancia para la infección humana por Trypanosoma cruzi en la región amazónica (Deane, et al., 1984; Coura, et al., 2006; Pinto, et al., 2008).

El primer caso humano de enfermedad de Chagas en la región amazónica fue relatado por Floch y Tasque (1941) en la Guyana Francesa y 
28 años después en Brasil por Shaw, et al. (1969). Posteriormente, prácticamente todos los países de la región amazónica informaron casos agudos de la enfermedad, aislados o en brotes epidémicos por transmisión oral (Aguilar, et al., 2007; Valente, et al., 1999; Pinto, et al., 2008).

Los riesgos de presentación endémica de la enfermedad de Chagas en la región amazónica, naturalmente de acuerdo con las peculiaridades de cada país, están relacionados con los siguientes factores:

1) El extenso reservorio de mamíferos silvestres infectados con $T$. cruzi, está representado en la amazonia brasilera por 33 especies, de seis órdenes: Marsupialia, Chiroptera, Rodentia, Edentada (Xenarthra), Carnivora e Primata.

2) De las 27 especies de triatominos reconocidos en la panamazonia, 16 que pertenecen a seis géneros, principalmente Rhodnius, ya fueron encontrados en la amazonia brasilera (Coura, et al., 2002; Junqueira, et al., 2005), 10 de las cuales estaban infectadas con $T$. cruzi.

3) Se han presentado diversos brotes de la enfermedad aguda de Chagas transmitida por la ingestión de alimentos contaminados con heces de triatominos, secreciones odoríferas o por la orina de marsupiales en la región, particularmente en Pará, Amapá, Maranhão y Acre (Valente, et al., 1999; Pinto, et al., 2008) y, más recientemente, en el estado del Amazonas.

4) La deforestación incontrolada en la región amazónica, con reducción y desplazamiento de los mamíferos silvestres, fuentes naturales de alimentación de los triatomínos, estimula la preadaptación y adaptación de los vectores al domicilio humano en busca de nuevas fuentes alimentarias.

5) La creciente migración de personas y de sus animales domésticos de regiones endémicas para la enfermedad de Chagas hacia la Amazonia, favorecida por la abertura de nuevas carreteras, construcción de hidroeléctricas, explotación de petróleo y gas natural, y otras actividades laborales (lícitas o ilícitas), puede promover una verdadera diseminación de la enfermedad hacia dicha región.

6) Finalmente, la falta de conocimientos y de demandas específicas para la vigilancia de la enfermedad de Chagas en la región amazónica, las dificultades de acceso y el predominio de actividades de explotación por parte de los nativos, como se mencionó anteriormente, constituyen no solamente un riesgo sino también una realidad del comportamiento localde la enfermedad.

La presencia de $R$. prolixus en el Orinoco es extremadamente preocupante para su transmisión a la región amazónica (Aguilar, et al., 2007), por tratarse de un vector adaptado al domicilio en varios países de Centroamérica y en algunos países de la región andina. Por otro lado, la comprobación de la domiciliación de $T$. maculata en el estado de Roraima, y posiblemente en el Estado de Tocantins en la amazonia brasilera, es de extrema gravedad pues esa especie es un vector importante en Venezuela.

\section{Enfermedad de Chagas en la amazonia} brasilera

La enfermedad de Chagas en la amazonia brasilera, de la misma forma que en la panamazonia como un todo, siempre se había considerado una enzootia de animales silvestres, hasta cuando Shaw, et al. (1969) describieron los cuatro primeros casos de infección humana por $T$. cruzi en Belém do Pará, en un pequeño brote probablemente por transmisión oral.

Más recientemente, Pinto, et al. (2008) estudiaron 233 casos agudos de la enfermedad observados de 1968 a 2005 en el Instituto Evandro Chagas de Pará, originarios de Pará, Amapá y Maranhão, la mayoría de ellos [78,5 \% (183/233)] ocurridos en brotes de probable transmisión oral y $21,5 \%$ (56/233) como casos agudos aislados. Inicialmente la autora había informado 430 casos de la enfermedad, observados en aquel Instituto, los cuales se redujeron a 233, ya que 197 no llenaban los criterios de casos agudos: aquéllos que tenían $T$. cruzi en el examen directo, en gota gruesa teñida o mediante métodos de concentración como Strout y QBC o IgM anti-T. cruzi.

Ésta es realmente la mayor casuística de casos agudos de la enfermedad de Chagas en la región amazónica. Además, la notificación de casos agudos en la amazonia brasilera ha venido aumentando de forma exponencial. La Secretaría de Vigilancia en Salud del Ministerio de la Salud, reportó 761 casos entre 1969 y 2008, de los cuales, $75 \%$ (568 casos) ocurrieron en el periodo de 2002 a 2008 (Brum-Soares, et al., 2010).

Una investigación serológica nacional realizada por la Superintendencia de Campañas (SUCAM) del Ministerio de Salud, entre 1975 y 1980 (Camargo, et al., 1984), reveló una prevalencia promedio para Brasil de 4,2 \%, con las tasas más altas observadas en los estados de Rio Grande 
do Sul (8,8 \%), Minas Gerais (8,8 \%), Goiás $(7,4$ $\%)$, Alagoas (6\%), Bahía (5,4\%), Paraná (4\%), Piauí (4\%), Paraíba (3,5\%), Pernambuco y Mato Grosso (2,8 \%). En la Amazonia, los dos estados con mayor prevalencia fueron Acre con (2,4\%), posiblemente debido a la migración de portadores de la infección chagásica del nordeste, y Amazonas $(1,88 \%)$; Pará presentó una prevalencia de apenas $0,5 \%$. Sin embargo, Silveira y Passos (1986) en un análisis más detallado por municipios de los estados amazónicos, identificaron "espacios" de infección chagásica por encima del promedio nacional. Entre ellos se destacan Colares, en el estado de Pará (5,1\%) y Barcelos, Novo Airão e Japurá, en el estado de Amazonas (6,3\%, 6,8 \% y $6,9 \%$, respectivamente).

Después de esta investigación nacional, se llevaron a cabo numerosos estudios sobre brotes agudos, informes de casos aislados y de prevalencia y morbilidad de la enfermedad de Chagas en la amazonia brasileira; entre ellos se destacan los de Valente, et al. (1999); Coura, et al. (1999, 2002); Pinto, et al., (2008); Albajar, et al. (2003); Junqueira, et al. (2005); Xavier, et al. (2006), y Brum-Soares, et al. (2010), entre otros.

En tres estudios serológicos preliminares que realizamos en la sede del municipio de Barcelos (Coura, et al., 1999), en 1991, 1993 y 1997, respectivamente, en una muestra de 2.254 residentes de aquel municipio amazónico, observamos una prevalencia promedio de $13 \%$; no obstante, los estudios serológicos posteriores con técnicas confirmatorias de inmunofluorescencia indirecta, ELISA convencional y recombinante, y Western blot (Tesa-blot), solamente pudimos confirmar como positivos de 2,8 a $5 \%$. Un estudio reciente que realizamos con inmunofluorescencia en papel de filtro en una muestra de 4.880 residentes en Barcelos, verificamos que 221 $(4,5 \%)$ tenían la serología fuertemente reactiva, considerada positiva, y 302 (6,2 \%) presentaron serología reactiva débil, considerada incierta. En este momento estamos recolectando sangre venosa de aquellos casos para confirmación serológica.

Como conclusión, podemos considerar que la enfermedad de Chagas en la región amazónica presenta tres perfiles:

1) infección enzoótica entre animales y vectores silvestres en toda la región;

2 antropozoonosis cuando el hombre invade el ecótopo silvestre y se infecta o cuando vectores y animales silvestres invaden el domicilio humano e infectan directamente al hombre o contaminan sus alimentos con heces y orina, $y$

3 enfermedad endémica profesional de poblaciones que hacen explotación vegetal, principalmente recolectoras de palma de chiquichiqui en áreas específicas (Coura, et al., 1999, 2002).

\section{Referencias}

1. Aguilar HM, Abad-Franch F, Dias JCP, Junqueira ACV, Coura JR. Chagas disease in the Amazon Region. Mem Inst Oswaldo Cruz. 2007;102(Suppl.1):47-55.

2. Albajar PV, Loredo SV, Terrazas MB, Coura JR. Miocardiopatia dilatada em pacientes com infecção chagásica crônica. Relato de dois casos fatais autóctones do Rio Negro, Estado do Amazonas. Rev Soc Bras Med Trop. 2003;36:401-7.

3. Brum-Soares LM, Xavier SS, Souza AS, BorgesPereira J, Ferreira JMBB, Costa IR, et al. Morbidade da doença de Chagas em pacientes autóctones da microrregião do Rio Negro, Amazonas. Rev Soc Bras Med Trop. 2010;43:170-7.

4. Camargo ME, Silva GR, Castilho EA, Silveira AC. Inquérito sorológico da prevalência da infecção chagásica no Brasil, 1975-1980. Rev Inst Med Trop São Paulo. 1984;26:192-204.

5. Coura JR. Transmissão da infecção chagásica por via oral na história natural da doença de Chagas. Rev Soc Bras Med Trop. 2006;39(Supl.IV):113-7.

6. Coura JR, Junqueira ACV, Boia MN, Fernandes O. Chagas disease: From bush to huts and houses. Is it the case of the Brazilian Amazon? Mem Inst Oswaldo Cruz. 1999;94(Suppl.I):379-84.

7. Coura JR, Junqueira ACV, Fernandes O, Miles MA. Emerging Chagas disease in Amazonian Brazil. Trends Parasitol. 2002;18:171-6.

8. Deane MP, Lenzi HL, Jansen AM. Trypanosoma cruzi: Vertebrate e invertebrate cycles in the same mammal host. Mem Inst Oswaldo Cruz. 1984;79:513-5.

9. Floch $\mathrm{H}$, Tasque P. Um cas de maladie de Chagas em Guyane Française. Bull Soc Path Exot. 1941;36:37.

10. Junqueira ACV, Albajar PV, Coura JR. Doença de Chagas na Amazônia brasileira. In: Coura JR. Dinâmica das doenças infecciosas e parasitárias. Rio de Janeiro: Ed. Guanabara Koogan; 2005. p. 595-601.

11. Pinto AYN, Valente AS, Valente VC, Ferreira AC, Coura JR. Fase aguda da doença de Chagas na Amazônia brasileira: estudo de 233 casos do Pará, Amapá e Maranhão observados entre 1988 e 2005. Rev Soc Bras Med Trop. 2008;41:602-14.

12. Shaw J, Lainson R, Fraiha $H$. Considerações sobre a epidemiologia dos primeiros casos autóctones da doença de Chagas registrados em Belém do Pará, Brasil. Rev Saúde Publ. 1969;3:153-7. 
13. Silveira AC, Passos ADC. Altos índices de prevalência sorológica de infecção chagásica em áreas da Amazônia. Rev Soc Bras Med Trop. 1986;19:45.

14. Valente SAS, Valente VC, Fraiha-Neto $H$. Considerations on the epidemiology and transmission of Chagas disease in Brazilian Amazon. Mem Inst Oswaldo Cruz. 1999;94(Suppl.I): 395-8.
15. Xavier SS, Souza AS, Albajar PV, Junqueira ACV, Boia MN, Coura JR. Cardiopatia chagásica crônica no Rio Negro, Estado do Amazonas. Relato de três novos casos autóctones comprovados por exames sorológicos, clínicos, radiográficos, eletro e ecocardiográficos. Rev Soc Bras Med Trop. 2006;36:211-6.

\title{
Enfermedades parasitarias en la región amazónica colombiana: nuevos descubrimientos
}

\author{
Iván Darío Vélez \\ Programa de Estudio y Control de Enfermedades Tropicales, Facultad de Medicina, \\ Universidad de Antioquia, Medellín, Colombia
}

Los estudios de campo realizados en las comunidades indígenas tikunas y cocamas de la cuenca del Amazonas en Colombia, permitieron detectar la alta prevalencia de enfermedades como mansonelosis, leishmaniasis y malaria, y otras infecciones por protozoos y helmintos intestinales.

Mansonella ozzardi está presente en más de $40 \%$ de la población tikuna, ocasionando pocos síntomas. La infección por Plasmodium, ya sea por $P$. falciparum, $P$. vivax o infección mixta, detectada por PCR, se presentaba en $32 \%$ de la población en forma asintomática.

Por otra parte, la infección por Leishmania se encontró directamente relacionada con las actividades de la comunidad. Aunque la prueba de Montenegro positiva, así como los casos activos e históricos, prevalecen en hombres adultos dedicados a actividades de cacería, dentro de las malocas y alrededor de ellas no se encontraron los flebotominos vectores; la baja prevalencia de la infección en mujeres y niños se explica por su comportamiento dentro dentro de las malocas y alrededor de ellas, donde no llegan los insectos vectores. También, se encontraron casos de leishmaniasis mucosa y dos casos de enfermedad de Chagas asintomáticos, pero con serología positiva para infección por Trypanosoma cruzi.

\section{Infección asintomática por Plasmodium spp. en la Amazonia: implicaciones para la eliminación de la malaria}

\author{
Martha Suárez-Mutis \\ Laboratório de Doenças Parasitárias, Instituto Oswaldo Cruz, Rio de Janeiro, RJ, Brasil
}

La malaria es una enfermedad esencialmente de áreas tropicales y subtropicales del planeta, y un grave problema de salud pública en más 100 países del mundo (WHO, 2010). La mayoría de los casos de malaria en el continente americano ocurren en la región amazónica. La transmisión de esta endemia es muy heterogénea; las áreas endémicas pueden ser clasificadas según los diferentes grados de la endemia y riesgo epidemiológico.

De la misma forma, la malaria es una enfermedad que tiene un amplio espectro clínico, que va desde la infección asintomática hasta las formas complicadas y la muerte. Aunque desde hace más de un siglo se reconoce la existencia de la infección asintomática por Plasmodium en áreas de malaria totalmente o muy endémicas, esta situación es relativamente nueva en el continente americano, considerado como un área medianamente o poco endémica. En los países africanos, donde se detectan individuos asintomáticos, es frecuente encontrar una parasitemia mayor que la observada en las áreas americanas con malaria. En este continente, los individuos asintomáticos suelen tener parasitemias muy bajas que pueden llegar hasta el nivel de infecciones subclíncas, detectadas solamente por la reacción en cadena de la polimerasa (PCR) y no por la gota gruesa convencional. La prevalencia varía entre 4,2 y 38,5 $\%$ cuando se usa la gota gruesa como método diagnóstico y, entre 10,6 y $64,8 \%$, cuando se utiliza la PCR.

En los últimos años se ha realizado una serie de estudios, con diferentes diseños epidemiológicos, que han permitido establecer la presencia de 
infección asintomática en varias áreas de la región amazónica. Usando la gota gruesa como método de diagnóstico, se han encontrado las siguientes prevalencias en Brasil: 14,3 \%, en mineros artesanales en Peixoto de Azevedo (Mato Grosso) en un estudio transversal sin seguimiento (Andrade, etal., 1995); 15,4\%, entre mineros en Apiacás (Mato Grosso) seguidos durante 15 días (Fontes, 2001); 38,5 y $4,9 \%$ en indígenas yanomami de la frontera en Brasil e Venezuela, respectivamente (Marcano, et al., 2004), y 4,1 a $16,9 \%$ en Portochuelo y Jiparaná (Rondonia) en un seguimiento de 60 días (Alves, et al., 2002). En Colombia, Suárez Mutis, et al. (2000) encontraron una prevalencia de 21,6 $\%$ entre indígenas yujup-maku en el río Apaporis (Amazonas), con un seguimiento de ocho días.

Mediante estudios con técnicas moleculares, se ha demostrado que la infección asintomática es más frecuente de lo que parecía. En Brasil, Scopel (2005), usando la PCR en las mismas muestras de Fontes (2001), encontró que $27,3 \%$ eran positivas para el plasmodio; en los estudios de Alves, et al. (2002) en Portochuelo y Ji-Paraná, la prevalencia varió entre 6,4 y 64,8\%; Ladeia-Andrade (2005) encontró una prevalencia de 31,3\% entre poblaciones ribereñas agrícolas en el parque Nacional del Jaú, río Negro, Brasil; Suárez-Mutis, et al. (2007) encontraron una variación entre 8.2 y 24 $\%$ en el río Padauiri (río Negro medio, Amazonas); en Vila Candeias (Rondonia), la prevalencia fue de 10,6 a 19,2 \% (Tada, et al., 2007) y, de 29,4 \%, en el estado del Acre, Brasil (Silva Nunes y Ferreira, 2007).

Branch, et al. (2005), en una localidad cercana a lquitos (Perú), detectaron una frecuencia de $59 \%$, y Roshavaran, et al. (2003) encontraron que una tercera parte de las gotas gruesas y 75 $\%$ de las PCR positivas ocurrían en individuos asintomáticos.

En el estudio de nuestro grupo en el río Negro medio, se demostró una asociación entre estacionalidad e infección asintomática. En la época de la estación seca hubo $5,5 \%$ de personas asintomáticas detectadas por la gota gruesa y $28,1 \%$ detectadas por la PCR, mientras que en la época de las lluvias se encontró $1,2 \%$ asintomáticos detectados por gota gruesa y $7,8 \%$ con la PCR. Con la malaria sucede lo contrario: la prevalencia de esta enfermedad es mayor en la época de las lluvias. Todas estas diferencias fueron estadísticamente significativas (Suárez-Mutis, 2007).

Los factores determinantes de la infección asintomática aún no están totalmente aclarados.
En áreas totalmente o muy endémicas de algunas regiones africanas, se sabe que los individuos que han sufrido múltiples episodios de malaria desarrollan inmunidad clínica; este tipo de inmunidad protege contra la enfermedad, es la primera que se desarrolla y parece ser específica para especie y fase, y más efectiva cuando hay una nueva reinfección con genotipos homólogos a aquellos experimentados con anterioridad (Snow y Marsh, 1998); además, es capaz de controlar la enfermedad y reducir la densidad de los parásitos. La inmunidad clínica, generalmente, se desarrolla lentamente durante la infancia y la adolescencia, y parece ser la responsable de la infección asintomática.

En América, poco se ha avanzado en el conocimiento de los factores determinantes de esta forma de infección por Plasmodium. Siendo así, es importante encontrar algunos "marcadores epidemiológicos" que permitan detectar infecciones asintomáticas en las diversas áreas palúdicas del continente. Los trabajos de Fontes (2001) mostraron que los mineros con más de 28 años de edad tenían mayores probabilidades de ser asintomáticos, si bien pudo haber cierto sesgo en esta muestra porque se trabajó sólo con población adulta. Marcano, et al. (2004) demostraron una menor prevalencia de malaria en individuos con más de 10 años de edad, en los cuales hubo una alta frecuencia de infección asintomática (38,5\%). Sin embargo, en el trabajo de Alves, et al. (2002) se muestra que la posibilidad de tener una infección asintomática fue 6,7 veces mayor en los adultos de más de 40 años, si se comparan con niños menores de 5 años, y al dividir el grupo entre menores de 15 años y mayores de 15 años, la probabilidad fue 5,8 veces mayor entre los adultos. Nuestros datos muestran que las personas mayores de 20 años tenían una probabilidad 1,79 veces mayor de tener infección asintomática, cuando se compararon con otros grupos de menor edad (Suárez-Mutis, 2007).

Fontes (2001) estableció otros factores condicionantes de importancia. Las personas que llevaban más tiempo viviendo en un área endémica, el número de meses después del primer episodio de malaria en la vida y el número de meses después del último episodio, estaban asociados a una mayor probabilidad de tener una infección asintomática. Los trabajos de Alves, et al. (2002) apuntan para hallazgos similares en el sentido de que las personas que hayan vivido durante más tiempo en la Amazonía, en localidades muy endémicas 
para malaria, tienen más probabilidades de ser asintomáticos.

Aunque la gota gruesa continúa siendo el método de referencia para el diagnóstico de malaria, no tiene la suficiente sensibilidad para la detección de portadores asintomáticos que se caracterizan por tener bajas parasitemias, ni siquiera al intentar aumentar la sensibilidad diagnóstica leyendo un mayor número de campos microscópicos (SuárezMutis y Coura, 2006). Laserson, et al. (1999) encontraron un promedio de 489 parásitos $/ \mathrm{mm}^{3}$ en pacientes con malaria, en comparación con 124 parásitos $/ \mathrm{mm}^{3}$ entre asintomáticos con infecciones por $P$. vivax. Alves et al. encontraron menos de 500 parásitos $/ \mathrm{mm}^{3}$ entre portadores asintomáticos.

A pesar de la baja parasitemia reportada entre los pacientes con infección asintomática en las Américas, su importancia reside en el hecho de que pueden estar haciendo parte de la cadena de transmisión de la enfermedad, sin ser detectados por los programas de control que sólo practican la gota gruesa a las personas con síntomas clínicos. Alves, et al. (2005) realizaron estudios de infección experimental, alimentando artificialmentemosquitos con sangre de pacientes. De los mosquitos alimentados con sangre de pacientes sintomáticos, $22 \%$ adquirieron posteriormente la infección, mientras que de los mosquitos alimentados con sangre de individuos asintomáticos, 1,2\% se infectaron.

En otras palabras, a pesar de que se ha demostrado que los portadores asintomáticos pueden infectar mosquitos, la eficiencia de la infección es muy baja cuando se compara con pacientes sintomáticos. Sin embargo, si en una localidad determinada $20 \%$ o más de las personas pueden estar asintomáticas en un momento determinado, el riesgo de infección puede ser alto. Estos estudios son aun preliminares y se realizaron con un pequeño número de muestras, por lo que es necesario hacer otros experimentos. Igualmente, es necesario el desarrollo de modelos matemáticos para establecer la probabilidad de transmisión de Plasmodium en estos individuos.

Con la perspectiva de la eliminación de la malaria como problema de salud pública mundial, es necesario hacer una intensa "búsqueda activa agresiva" (Macauley, 2005) para detectar los individuos con infección asintomática. El reto es mucho mayor en los países americanos, en los cuales $P$. vivax es el principal parásito implicado en la infección, pues cuando una persona comienza a tener síntomas que llevan al diagnóstico, ya está transmitiendo la enfermedad, pues los gametocitos se producen desde antes de iniciarse la sintomatología.

Por otro lado, es necesario continuar la búsqueda de "marcadores epidemiológicos" para la detección de áreas con presencia de portadores asintomáticos. Los resultados de los estudios sugieren que los siguientes hallazgos pueden ser necesarios para detectar individuos con infección asintomática: áreas endémicas con altos, y sostenidos, niveles de transmisión, por lo menos, en los cinco años anteriores, adultos que hayan tenido varios episodios previos de malaria, presencia de un vector eficiente, poblaciones tradicionales que hayan vivido en áreas endémicas durante largo tiempo, cambios en el patrón epidemiológico de la enfermedad, etc.

\section{Bibliografía}

1. Alves FP, Durlacher RR, Menezes MJ, Krieger H, Silva LHP, Camargo EP. High prevalence of asymptomatic Plasmodium vivax and Plasmodium falciparum infections in native Amazonian population. Am J Trop Med Hyg. 2002;66:641-8.

2. Alves FP, Gil LH, Marrelli MT, Ribolla PEM, Camargo EP, Silva LHP. Asymptomatic carriers of Plasmodium spp. as infection source for malaria vector mosquitoes in the Brazilian Amazon. $\mathrm{J}$ Mel Entomology. 2005;42:777-9.

3. Andrade ALSS, Martelli CMT, Oliviera RM, Arias JR, Zicker F, Pang L. High prevalence of asymptomatic malaria in Gold Mining areas in Brazil. Clin Infect Dis. 1995;20:467.

4. Branch OL, Casapia WM, Gamboa DV, Hernandez JN, Alava FF, Roncal N, et al. Clustered local transmission and asymptomatic Plasmodium falciparum and Plasmodium vivax malaria infections in a recently emergen hypoendemic Peruvian Amazon community. Malaria J. 2005;4:27-42.

5. Fontes CJF. Epidemiologia da malaria e fatores associados à infecção assintomática por Plasmodium em uma população de garimpeiros da amazonia brasileira (Mato Grosso, 1996) (tesis). Belo Horizonte: Universidade Federal de Minas Gerais; 2005.

6. Ladeia-Andrade S. Aspectos epidemiológicos da malária no Parque Nacional do Jaú, Amazonas, Brasil (tesis). Rio de Janeiro: Instituto Oswaldo Cruz, 2005.

7. Macauley C. Aggressive active case detection: A malaria control strategy based on the Brazilian model. Soc Sci Med. 2005;60:563-73.

8. Marcano TJ, Morgado A, Tosta CE, Coura JR. Crosssectional study defines difference in malaria morbidity in two Yanomami communities on Amazonian boundary between Brazil and Venezuela. Mem Inst Osw Cruz. 2005;99:369-76.

9. Scopel KKG, Fontes CFJ, Ferreira MU, Braga E. Plasmodium falciparum: IgG subclasse antibody 
response to merozoite surface protein-1 among Amazonian gold miners, in relation to infection status and disease expression. Exp Parasitol. 2005;109:12434.

10. Silva-Nunes M, Ferreira MU. Clinical spectrum of uncomplicated malaria in semi-immune Amazonians: Beyond the "symptomatic" Vs. "asymptomatic" dichotomy. Mem Ins Oswaldo Cruz. 2007;102:341-7.

11. Suárez-Mutis MC. Epidemiologia da malária em comunidades do rio Padauiri, médio rio Negro, uma área de extrativismo vegetal da piaçaba no Estado do Amazonas, Brasil (tesis). Rio de Janeiro: Instituto Oswaldo Cruz; 2007.

12. Suárez-Mutis MC, Bonilla MP, Blandón ME. Diagnosis of the health situation of the Yujup-Maku, a seminomadic indigenous group of the Colombian
Amazonas. Abstracts, XVth International Congress for Tropical Medicine and Hygiene and Malaria, Cartagena, 2000.

13. Suárez-Mutis MC, Coura JR. Evaluation of the thick smear in a field condition in a malaria endemic area in the middle region or Rio Negro, Amazon. Rev Soc Bras Med Trop. 2006;39:495-7.

14. Tada MS, Marques RP, Mesquita E, Martha RCD, Rodrigues JÁ, Costa JDN, et al. Urban malaria in the Brazilian Western Amazon Region I. High prevalence of asymptomatic carriers in an urban riverside district is associated with a high level of clinical malaria. Mem Inst Oswaldo Cruz. 2007;102:263-9.

15. WHO 2010. World Malaria Report 2010. Geneva; WHO. 